\title{
Ruptured primary ovarian ectopic pregnancy: a case series
}

\author{
Neha Singh*, Meenal Sarmalkar, Madhuri Mehendale, Arun Nayak
}

\begin{abstract}
Department of Obstetrics and Gynaecology, Lokmanya Tilak Municipal Medical College and General Hospital, Sion,
\end{abstract} Mumbai, Maharashtra, India

Received: 18 August 2016

Accepted: 15 September 2016

\section{*Correspondence:}

Dr. Neha Singh,

E-mail: dr.nick87@gmail.com

Copyright: (c) the author(s), publisher and licensee Medip Academy. This is an open-access article distributed under the terms of the Creative Commons Attribution Non-Commercial License, which permits unrestricted non-commercial use, distribution, and reproduction in any medium, provided the original work is properly cited.

\begin{abstract}
Ovarian ectopic pregnancy (OEP) is one of the rarest forms of nontubal ectopic pregnancies. Less than $1 \%$ of ectopic pregnancies are primary OEP. Exact etio-pathogenesis and predisposing factors for OEP are not yet well understood. Review of literature suggests its possible association with conditions like tubal sterilization, intrauterine contraceptive device (IUCD), IVF-ET in patients with bilateral salpingectomy etc. Clinical and radiological presentation often mimics like tubal ectopic pregnancy, making preoperative diagnosis difficult for clinicians. Thus, correct diagnosis can be made only intraoperatively using Speigelberg's criteria followed by histopathological confirmation. Treatment includes wedge resection of affected ovarian tissue or salpingo-oophorectomy in selected cases. We present a case series of primary ruptured ovarian ectopic pregnancy with no identifiable risk factors with inconclusive radiological diagnosis.
\end{abstract}

Keywords: Ectopic pregnancy, Ovarian ectopic pregnancy, Corpus luteal hematoma, Surgical management of ovarian ectopic pregnancy, Ovarian wedge resection, Salpingo-oophorectomy

\section{INTRODUCTION}

Approximately $2 \%$ of all pregnancies are ectopic pregnancies with $97 \%$ incidence of tubal ectopic pregnancy. Non-tubal ectopic pregnancies include ovarian, cervical, scar and cornual pregnancies. Ovarian ectopic pregnancy is an uncommon form of ectopic pregnancy which accounts for approximately $3 \%$ of all ectopic pregnancies. ${ }^{1}$ Majority of patients present clinically with nonspecific signs and symptoms with varying degrees of haemorrhagic shock secondary to rupture of ovarian ectopic leading to intraperitoneal haemorrhage. Preoperative evaluation with serum beta HCG levels and high resolution transvaginal sonography is useful in making presumptive diagnosis with limitations being inability to distinguish between tubal and ovarian ectopic pregnancy. Unlike tubal ectopic pregnancy, OEP has not been associated with risk factors predisposing for tubal ectopic like infertility and pelvic inflammatory diseases. Review of literature suggests its possible association with intrauterine devices and IVF-ET following bilateral salpingectomy. More often, patients present to hospital after rupture of ectopic pregnancy with haemodynamic instability or shock. ${ }^{2}$ We present here two interesting cases of ruptured primary ovarian ectopic pregnancies with no obvious risk factors.

\section{CASE REPORT}

\section{Case 1}

25 year old G2P1L1 with six weeks amenorrhea and positive UPT status came in emergency room with chief complaints of bleeding per vaginum since 5 days and pain in abdomen since 10 hours. In her obstetric history, she had full term vaginal delivery 4 years back. In present pregnancy, she conceived spontaneously. She did not give any history of major medical or surgical illnesses in past. She did not have any history of abdominal surgeries, PID, Tuberculosis. Her menstrual cycles were regular. On 
clinical examination, she had pallor with tachycardia. On per abdomen examination, generalized distension and tenderness in right iliac fossa were noted with guarding. On per speculum examination, cervix and vagina were healthy with altered blood stained discharge coming through OS.

On bimanual examination, uterus was bulky and anteverted with bilateral forniceal tenderness. No mass could be felt through fornices. All pre-operative investigations including complete hemogram, renal \& liver function tests were sent. On ultrasound, there was no evidence of intrauterine gestational sac with an irregular right adnexal hypoechoic mass of $7.3 \times 4.3 \times 3.2$ $\mathrm{cm}$ not seen separate from right ovary with hemoperitoneum with normal left ovary, suggestive of right ruptured tubal ectopic pregnancy. All investigations were within normal limits. Blood availability was confirmed. Patient was taken for emergency exploratory laparotomy. Intraoperatively, hemoperitoneum with right ovarian hemorrhagic mass of approximately $5 \times 5 \mathrm{~cm}$ with active bleeding from site of rupture was noted (Figure 1).

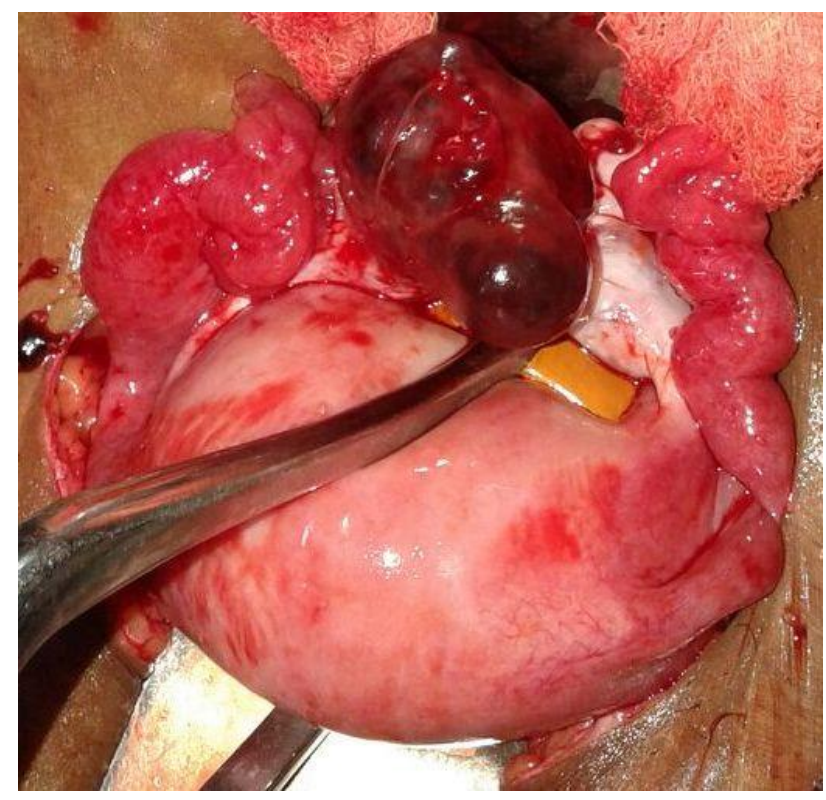

Figure 1: Right ovarian ectopic pregnancy.

Uterus was bulky with normal left fallopian tube and left ovary. Right fallopian tube was oedematous without any localized swelling or oozing from its fimbrial end. There was no evidence of adhesion, tubercles, endometriosis or any other pelvic pathology. Provisional diagnosis of ovarian ectopic pregnancy was made based upon intraoperative findings with possibility of right corpus luteal hematoma. In view of extensive ovarian tissue involvement and uncontrolled bleeding after wedge resection, right salpingo-oophorectomy was done and sample sent for histopathological examination. Patient was transfused blood intraoperatively. Patient had uneventful postoperative course and discharged on day 7 of surgery. Histopathological findings were consistent with diagnosis of primary ovarian ectopic pregnancy in view of presence of chorionic tissue within ovarian stroma (Figure 2) with absence of chorionic tissue in fallopian tube.

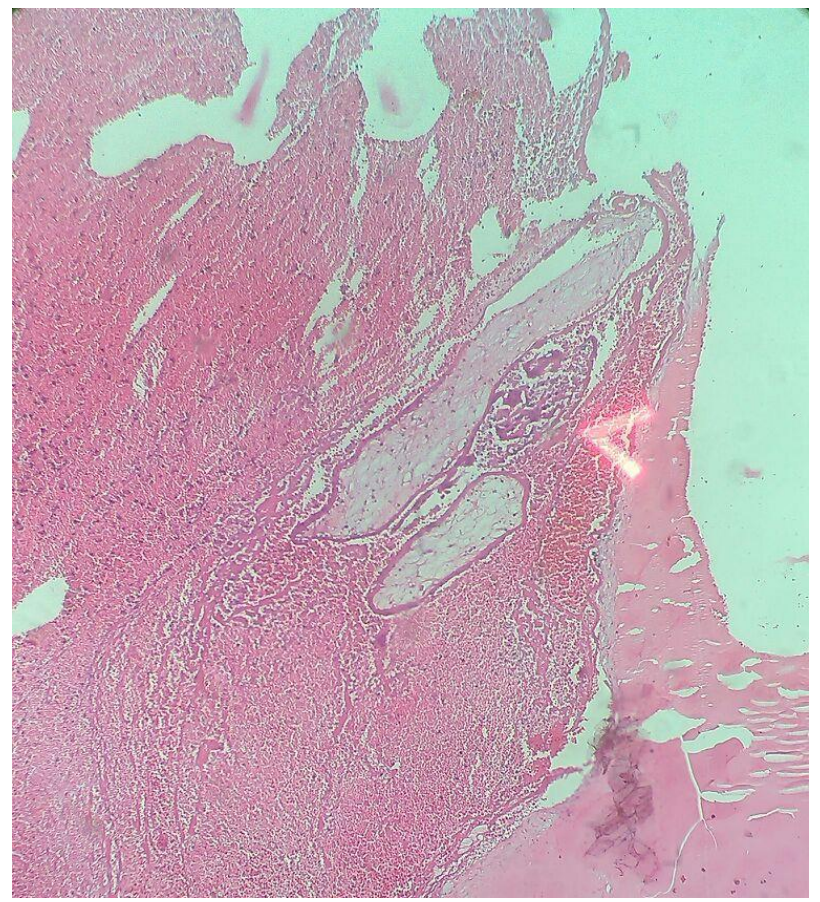

Figure 2: Histological picture of ovarian mass with arrow showing chorionic tissue within ovarian stroma.

\section{Case 2}

27 years old G3P2L2 with 5 weeks amenorrhoea with positive UPT was attended in emergency in view of pain in abdomen since 1 week. She had previous two normal vaginal deliveries with last child birth one and half years back. Her past medical and surgical history was not significant .She had no history of any menstrual irregularity. She did not have any obvious risk factor for ectopic pregnancy like use of contraceptive device. On clinical examination, she had pallor with tachycardia with abdominal tenderness and guarding. On per speculum examination, altered blood stained discharge noted through OS. On per vaginal examination, uterus was bulky and anteverted with right forniceal tenderness without any palpable mass. Ultrasound findings were suggestive of bulky uterus with no intrauterine pregnancy and a heterogeneous hyperechoic lesion of $2.8 \times 2.5 \times 3 \mathrm{~cm}$ containing $7 \times 6 \mathrm{~mm}$ irregular gestational sac like structure in right adnexa with hemoperitoneum suggestive of ruptured ectopic pregnancy. Left ovarian hemorrhagic cyst of $3.3 \times 2.5 \times 3 \mathrm{~cm}$ size was noted with bilateral bulky fallopian tubes. Emergency exploratory laparotomy was done. Intraoperatively, hemoperitoneum with bulky uterus and left ovarian hemorrhagic cyst of $3 \times 3 \mathrm{~cm}$ with right ovarian ruptured ectopic pregnancy satisfying Speigelberg's criteria with bilateral normal fallopian tubes were noted. There was no evidence of any pelvic pathology. Provisional diagnosis of ovarian ectopic 
pregnancy was made based upon ultrasound and intraoperative findings with possibility of right corpus luteal hematoma. Right ovarian wedge resection was done followed by ovarian reconstruction and left ovarian cyst aspiration. Patient was transfused blood intraoperatively. Patient had uneventful postoperative course and discharged on day 7 of surgery. Histopathological findings were consistent with diagnosis of ovarian ectopic pregnancy.

\section{DISCUSSION}

Due to rarity of ovarian ectopic pregnancy, there is lack of enough evidence regarding its etiopathogenesis and management. This condition seems to be strongly associated with intrauterine devices. Preoperative evaluation includes serum beta HCG levels and TVS.

OEP can be classified as primary and secondary OEP. Primary OEP results from ovulatory dysfunction whereas secondary OEP results from secondary implantation in ovarian stromal tissue after tubal abortion or perforation. Begum et al published a case series of three patients with ovarian pregnancy. Two patients had history of contraceptive intrauterine device insertion. ${ }^{3}$

In a study by Christine et al, ultrasonographic findings of ovarian ectopic pregnancies were evaluated. Six cases in the period of 13 years were studied with menstrual ages ranging from 6-9 weeks. A wide echogenic ring with an internal echo lucent area was noted in 5 cases and in one case, there was presence of yolk sac and another case had fetal cardiac activity. Intraoperatively, all ovarian pregnancies had similar appearance like hemorrhagic ovarian cyst. In the patient in whom echogenic ring was not seen, had ruptured ectopic pregnancy. They concluded that ovarian pregnancies usually appeared on or within ovary as a cyst with a wide echogenic ring. A yolk sac or embryo was less commonly seen. ${ }^{1,2}$

Speigelberg's criteria $^{1}$ for intraoperative diagnosis of OEP include:

- Intact fallopian tubes including fimbria, separate from the ovary

- Ectopic pregnancy occupying normal position of the ovary

- Ectopic gestational sac must be attached to uterus through the utero-ovarian ligament

- Presence of ovarian tissue in the wall of gestational sac in specimen.

Melcer et al conducted a retrospective study over the period of 43 years in an institute to assess the clinical manifestations, management and post-surgical outcome. Forty six out of 2610 ectopic pregnancies were primary OEP $(1.8 \%)$. Majority had history of IUD insertion. All had nonspecific pelvic USG findings pointing towards ectopic pregnancy but inconclusive for diagnosis of OEP. These findings included empty uterine cavity or IUD in situ, complex adnexal mass, free fluid in pouch of Douglas. All patients were managed surgically by either laparotomy or laparoscopy. They concluded that ovarian pregnancy continues to be a diagnostic challenge and associated with high incidence of circulatory collapse and hemoperitoneum requiring blood transfusions and longer hospitalization. $^{4}$

Another retrospective cross-sectional study was done by Goyal et al over duration of ten years with aims to find incidence, risk factors, role of USG in preoperative diagnosis, feasibility of conservative management with medical method or minimal invasive surgery in a developing country like India. They found $4.9 \%$ incidence of OEP of all ectopic (14/523). Preoperative diagnosis of OEP was made in 2 cases. All cases were managed surgically with laparotomy with wedge resection of ovary and 2 cases required oophorectomy. They concluded that USG can detect OEP in unruptured state but it is of limited role in ruptured cases. Medical management is not feasible in most of the cases as patients present after rupture of OEP. ${ }^{5}$

In most of the cases, OEP is managed surgically due to majority of patients being brought with ruptured pregnancy and hemodynamic instability secondary to early rupture of friable nature and increased vascularity of OEP and radiological diagnostic dilemmas. There is lack of enough evidence on conservative nonsurgical management options. These options include methotrexate therapy (single or multiple doses) either given locally or systemic, potassium chloride, and hyperosmolar glucose. Allison et al reported an interested case of heterotopic OEP in a 31 year primigravida with $63 / 7$ weeks with primary infertility conceived after treatment. She was managed successfully with $50 \%$ hypertonic glucose injected transvaginally into ectopic gestational sac with intrauterine pregnancy continued till term with good perinatal outcome. ${ }^{6}$

\section{CONCLUSION}

With the availability of sensitive and specific radioimmunoassay for human chorionic gonadotropin, high resolution transvaginal ultrasound, and laparoscopy, early diagnosis of ectopic pregnancy has become possible. But, making preoperative diagnosis of OEP is still challenging due to its similar appearance to corpus luteal hematoma or hemorrhagic cyst of ovary before rupture and to ruptured tubal ectopic pregnancy after rupture. Therefore, better investigations and expertise in imaging techniques are urgently required for early diagnosis to gain time for conservative nonsurgical management to reduce morbidity secondary to blood loss and surgery.

\section{Funding: No funding sources \\ Conflict of interest: None declared \\ Ethical approval: Not required}




\section{REFERENCES}

1. Comstock C, Huston K, Lee W. The ultrasonographic appearance of ovarian ectopic pregnancies. Obstet Gynecol. 2005;105:42-5.

2. Casikar I, Reid S, Condous G. Ectopic pregnancy: ultrasound diagnosis in modern management. Clin obstet Gynecol. 2012;55(2):402-9

3. Begum J, Pallavee P, Samal S. Diagnostic dilemma in ovarian pregnancy: A case series. J Clin Diagn Res. 2015;9(4):QR01-3.

4. Melcer Y, Smorgick N, Vaknin Z, Mendlovic S, Raziel A, Maymon R. Primary ovarian pregnancy:
43 years' experience in a single institute and still a medical challenge. Isr Med Assoc J. 2015;17:687-90.

5. Goyal L, Tondon R, Goel P, Sehgal A. Ovarian ectopic pregnancy: A 10 years' experience and review of literature. Iran J Reprod Med. 2014;12(12):825-30.

6. Allison J, Aubuchon M, Leasure J, Schust D. Hyperosmolar glucose injection for the treatment of heterotopic ovarian pregnancy. Obstet Gynecol. 2012;120:449-52.

7. Madhu YC, Harish K. Complete resection of a giant ovarian tumour: a case report. Gynaecologic Oncology Case Reports. 2013;6:4-6.

Cite this article as: Singh N, Sarmalkar M, Mehendale M, Nayak A. Ruptured primary ovarian ectopic pregnancy: a case series. Int J Reprod Contracept Obstet Gynecol 2016;5: 3641-4. 